\title{
Germanica
}

\section{Entre quête et enquête : passé et présent dans la trilogie « Selb » de Bernhard Schlink}

Zwischen Spuren- und Selbstsuche: Gegenwart und Vergangenheit in Bernhard Schlinks Kriminalromanen "Selb“

Between investigation and quest :past and present in Bernhard Schlink's

detective novels "Selb"

Ingeborg Rabenstein-Michel

\section{OpenEdition}

\section{Journals}

Édition électronique

URL : http://journals.openedition.org/germanica/3187

DOI : 10.4000/germanica.3187

ISSN : 2107-0784

\section{Éditeur}

Université de Lille

\section{Édition imprimée}

Date de publication : 30 septembre 2016

Pagination : 81-93

ISBN : 9782913857377

ISSN : 0984-2632

\section{Référence électronique}

Ingeborg Rabenstein-Michel, « Entre quête et enquête : passé et présent dans la trilogie « Selb » de Bernhard Schlink », Germanica [En ligne], 58 | 2016, mis en ligne le 30 septembre 2018, consulté le 06 octobre 2020. URL : http://journals.openedition.org/germanica/3187 ; DOI : https://doi.org/10.4000/ germanica.3187 


\title{
Entre quête et enquête : passé et présent dans la trilogie «Selb» de Bernhard Schlink
}

\author{
Ingeborg RABENSTEIN-MICHEL \\ Université Claude Bernard-Lyon 1
}

Bernhard Schlink, juge, professeur de droit public et de philosophie du droit, connu pour le succès international de son roman Der Vorleser (Le Liseur, 1995), avait commencé sa carrière d'écrivain en publiant des romans policiers dont l'un des premiers, Die gordische Schleife (Le næud gordien, 1988), obtient le Prix Glauser en 1989. Une année plus tôt était paru le premier volet de ce qui allait devenir la « trilogie Selb »1 qui nous semble représenter une approche assez particulière du genre du « polar », quelle que soit sa définition (roman d'énigme, roman noir, thriller etc.). Car au-delà du schéma traditionnel de l'affaire criminelle à résoudre, ces trois romans policiers construisent un univers fictionnel ${ }^{2}$

1. - Selbs Justiz (1987), avec Walter Popp. Titre français : Brouillard sur Mannheim. Traduction de Martin Ziegler, revue par Olivier Mannoni, Gallimard, 1997, Selbs Betrug (1992), Un hiver à Mannheim. Traduction de Patrick Kermann, revue par Oliver Mannoni, Gallimard, 2000 et Selbs Mord (2001), La fin de Selb. Traduction de Martin Ziegler, revue par Olivier Mannoni, Gallimard 2003. Les citations renvoient aux versions françaises, abrégées Brouillard, Hiver et Fin.

2. - Dans leur livre consacré au roman policier, Boileau et Narcejac (Le roman policier, Payot, 1964) considèrent que celui-ci est « d'abord une fiction, avant tout un roman » (p. 9). On peut donc parler « de l'univers romanesque de Patricia Highsmith, sans ridicule » (p. 216), affirmation que ne réfutera pas cette auteure qui se décrit

GERMANICA, 2016, LVIII, pp. 81 à 93. 
qui mêle intimement le passé et le présent de son détective - et de l'Allemagne.

Le roman policier a été théorisé non seulement par nombre de ses auteurs, mais très tôt par des spécialistes de la littérature et par des sociologues à l'instar de Siegfried Kracauer, disciple de Georg Simmel dont il avait suivi les cours de philosophie et de sociologie, qui a été l'un des premiers à rédiger entre 1922 et 1925 un « traité philosophique » (soustitre du texte) intitulé Der Detektiv-Roman ${ }^{3}$, publié à titre posthume et traduit en 1981 par Rainer et Geneviève Rochlitz pour la collection « critique de la politique » des Éditions Payot. Kracauer marchait alors sur les pas d'Ernst Bloch qui avait publié pour sa part un texte intitulé Philosophische Ansicht des Detektivromans ${ }^{4}$ (Considérations philosophiques du roman policier). Philosophique, politique, et, plus tard, « mythologique » comme le veut le titre de l'ouvrage de Francis Lacassin 5 : le roman policier est rapidement perçu comme un genre plus complexe qu'il n'y paraît à première vue et qui dépasse le simple objectif de distraire son lecteur, que Bloch décrivait confortablement installé dans son fauteuil, un verre et la pipe à la main...6.

Kracauer constatait très justement que le roman policier « n'est plus depuis longtemps une mixture [...], mais un genre stylistique bien défini. » (p. 27). Les exemples (Poe, Conan Doyle, Leroux, Leblanc...) sur lesquels il s'appuie relevaient bien sûr essentiellement du roman à problème ou d'énigme qui deviendra le classique du genre avec les " grandes dames » de la littérature policière du $\mathrm{XX}^{\mathrm{e}}$ siècle, Agatha Christie en tête. Inattendu mais intéressant le rapprochement avec le conte de fées qu'il opère à la fin de sa réflexion: le happy-end fait en effet partie de l'outillage de la plupart des textes de cette catégorie. Les policiers d'Agatha Christie sont en cela exemplaires : une fois la solution de l'énigme annoncée par le détective omniscient, le retour non seulement à l'ordre mais au bonheur se fait sans heurts, les plus effroyables problèmes de famille déballés au cours de l'enquête sont en fin de compte (de conte ?) réglés et oubliés sans laisser ni trace ni séquelles et le monde continue de tourner avec juste quelques personnages en moins : très souvent il s'agissait d'ailleurs des plus « dispensables »...Dans ces

prioritairement comme une romancière dans son livre L'art du suspense, mode d'emploi (Calman-Lévy, 1987), et parfaitement acceptable pour Schlink.

3. - In : Schriften I, Suhrkamp, Frankfurt/Main, 1971.

4. - In : Jochen Vogt (Hrsg.), Der Kriminalroman. Zur Theorie und Geschichte einer Gattung Bd I. UTB, Fink, München 1971, p. 38-51. Bloch avait par ailleurs consacré en 1928 un essai intitulé « Ludwigshafen-Mannheim » à sa ville natale, Mannheim, où se déroulent pour l'essentiel les enquêtes de Selb.

5. - Mythologie du roman policier, première édition 1974, Union Générale d'Editions.

6. - Bloch cité par Vogt, op. cit., p. 38. 
« whodunnit », le crime n'a en vérité pas d'autre réalité que de fournir

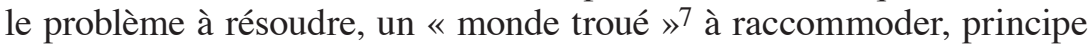
que l'on pourrait rapprocher de cette « insalubrité du monde » devenue cependant impossible à éradiquer que Selb évoque dans sa première enquête (Brouillard, p. 102). La solution/happy-end disparaît ainsi dans les policiers récents ${ }^{8}:$ il est devenu difficile de sauver le monde.

Les définitions du roman policier sont nombreuses et variées. Le dénominateur commun, comme le soulignent Boileau et Narcejac ${ }^{9}$, est cependant toujours l'enquête menée par un enquêteur. Du classique roman d'énigme à la " détective novel » urbaine à l'américaine ${ }^{10}$, du crime parfait ${ }^{11}$ au crime crapuleux, de l'inscription très documentée, voire érudite dans des époques révolues (la Chine ancienne pour le sinologue Van Gulik, l'Angleterre du XII siècle chez Ellis Peters, l'époque victorienne chez Anne Perry, la Vienne de Freud et de l'antisémitisme montant au tournant du $\mathrm{XX}^{\mathrm{e}}$ siècle chez Frank Tallis, etc.) aux enquêtes « tranquilles »d'un Maigret, le schéma de base est immuable : problème - enquête - solution. Les policiers de Schlink ne font pas exception à cette règle, tout en y apportant des éléments nouveaux.

\section{Le roman policier selon Schlink}

Les trois enquêtes de Selb contiennent une bonne dose de critique sociale telle que Maj Sjöwall et Per Wahlöö l'avaient introduite dans le roman policier avec la série des dix enquêtes du commissaire Martin Beck parues entre 1968 et 1977. Le crime y révélait les failles et défaillances d'une société considérant voire transformant chacun en un

7. - Pierre Bayard, Le Chien des Baskerville, Paris, Éditions de Minuit, 2008, p. 67-68. Bayard avait aussi analysé Le meurtre de Roger Ackroyd d'Agatha Christie (Éditions de Minuit, 1998) où Christie met à mal les vingt règles du roman policier édictées par S.S. Van Dine en 1928, dont le fait de « dissimuler le meurtrier sous la première personne du récit » (Roland Barthes, Le Degré zéro de l'écriture, Paris, Le Seuil, 1953, p. 28-29). Et Barthes poursuit : « Le lecteur cherchait l'assassin derrière tous les 'il' de l'intrigue : il était sous le "je"»...

8. - Récents ou « postmodernes »? À ce sujet Alida Bremer, Kriminalistische Dekonstruktion. Zur Poetik der postmodernen Kriminalromane, Würzburg, Königshausen \& Neumann, 1999.

9. - Op.cit., page 8.

10. - Lacassin fixe la naissance du roman noir « à l'américaine » à la fin des années vingt, avec les premières œuvres de Dashiell Hammet, Raymond Chandler, William Irish... Le roman policier devient alors « un phénomène urbain et quotidien » (Lacassin, p. 14), à la forte charge de violence purificatrice.

11. - Voire « artistique »? Facile chez Raymond Chandler (« The Simple Art of Murder », in: Atlantic Monthly, 1944) ou revendiquant le statut d'œuvre (cf. Thomas de Quincey dans On Murder Considered as one of the Fine Arts, 1827. Plusieurs traductions françaises dont de Pierre Leyris, Gallimard, 2002). 
criminel en puissance ${ }^{12}$ : la recherche du coupable s'y doublait d'une réflexion sur la manière dont un système produit sa criminalité. Les analyses et commentaires personnels de plus en plus désabusés que Sjöwall et Wahlöo attribuent à leur commissaire se retrouvent aussi chez Schlink qui ménage régulièrement des césures où les problèmes qui préoccupent Selb sont discutés ${ }^{13}$. Celui-ci, résolument atypique ${ }^{14}$ ne serait-ce que par son âge (soixante-huit ans au moment de sa première enquête) fréquente un cercle d'amis qui le secondent dans ses réflexions. Selb, veuf vivant seul avec son chat Turbo, est propriétaire d'une agence installée dans un ancien bureau de tabac. Ses affaires marchent plutôt bien et lui permettent même certaines années de réaliser ses objectifs financiers en quelques mois seulement. Elles ralentiront cependant dans le troisième roman ( $\mathrm{La}$ fin de Selb). Le détective privé a dorénavant dépassé les soixante-dix ans ${ }^{15}$, il est fatigué. Plusieurs infarctus mettront d'ailleurs (presque) fin à ses activités, et à sa vie.

Le nom Selb relie les trois titres comme cela a pu être le cas pour d'autres séries policières, de Father Brown (G.K.Chesterton) au commissaire Brunetti de Donna Leon en passant par Maigret (Georges Simenon). Elément fédérateur, la cristallisation forte autour du personnage de l'enquêteur (amateur, policier, détective privé etc.) est souvent une promesse de succès : le lecteur/voyeur suit alors en parallèle l'enquête et l'évolution propre au personnage central ${ }^{16}$. Dans la trilogie de Schlink, les titres

12. - Un point de vue cependant déjà exprimé, par exemple, par Agatha Christie. Rappelons que Christie clôturera la série consacrée à son détective belge en 1975 avec deux meurtriers inattendus : l'un involontaire, le capitaine Hastings, l'autre intentionnel, Poirot lui-même (cf. Curtain : Poirot's Last Case).

13. - Lors du suicide de l'un des suspects et finalement faux coupable, Selb (se) pose ainsi la question de la relation de cause à effet : " Du point de vue juridique, je ne suis pas coupable. C'est le problème humain qui m'intéresse » (Brouillard, p. 71).

14. - «Vous ne correspondez pas à l'image que je me suis faite d'un détective privé », constate l'un des personnages de la trilogie. "Ce n'est pas la première fois que j'entendais cette réflexion. Je sais aujourd'hui comment les gens s'imaginent un détective privé. Pas seulement plus jeune. Vous devriez me voir en imper ! » réplique Selb en faisant allusion au détective « hard-boiled » à la Humphrey Bogart dans, par exemple, l'adaptation cinématographique du Faucon Maltais (Brouillard, p. 21).

15. - Si l'on tient compte des soixante-huit ans de Selb en 1987, il devrait même être âgé de quatre-vingt-deux ans à la parution du dernier volume en 2001.

16. - Nous apprenons que Selb conduit une vieille Opel Kadett, aime la musique classique, Udo Jürgens et Hildegard Knef. Barry Lindon est son film préféré, il fume des Sweet Afton, a une préférence pour la Sambuca et un cocktail appelé «l'aviateur » (Campari, jus de pamplemousse, champagne). Il a ses habitudes dans le «Kleiner Rosengarten » où il fait régulièrement son numéro avec le serveur Giovanni. Il lit et relit Der grüne Heinrich (Gottfried Keller, 1854), passe ses vacances en Grèce et aime jouer aux échecs avec ses amis. Il décore son arbre de Noël avec des boîtes de sardines... Dans le premier volume, il entame une liaison avec Brigitte, quadragénaire stérilisée et pourtant mère d'un petit garçon, qui deviendra sa compagne. 
ont cependant la particularité supplémentaire de former, avec le nom du détective qui y paraît systématiquement, des substantifs composés qui présentent juste un léger défaut grammatical, le « $\mathrm{t}$ » manquant... Schlink joue ainsi avec un réseau significatif qui nous mène, en allemand, de la décision de Selb de faire justice lui-même (Selbs-t-justiz) au suicide (Selbs-t-mord), en passant par la prise de conscience des jeux de dupes dont il est victime, ou sont victimes les autres (Selbs-t-betrug). Ce dispositif terminologique, difficile à rendre en français ${ }^{17}$, a une fonction quasi programmatique et annonce la complexité des enquêtes, et le positionnement particulier du détective dans les trois romans.

\section{Autopsie d'une enquête}

Les enquêtes de Selb sont a priori parfaitement inscrites dans le présent avec des affaires de sabotage industriel, d'escroquerie à l'assurance, de blanchiment d'argent, de terrorisme, de détournements financiers sur fond de réunification allemande etc. Elles se complexifient en avançant systématiquement par imbrication. Dans chaque volet de la trilogie, l'affaire initiale fournit des indices insoupçonnés à des enquêtes secondaires qui peuvent se révéler l'affaire principale - et touchant de près l'enquêteur - à leur tour puisqu'immanquablement reliées au passé/à son passé allemands. Les différentes étapes de Brouillard sur Mannheim permettent d'illustrer ce principe :

Ferdinand Korten, directeur général de la Société Rhénane de Chimie (Rheinische Chemie Werke / RCW) et beau-frère de Selb, fait appel à ce dernier pour résoudre une affaire d'intrusion malveillante dans son système informatique. Selb identifie assez vite un possible suspect qui se suicide en laissant une lettre qui semble établir sa culpabilité. La RCW est contente, l'affaire est close, mais Selb n'est pas satisfait. Il continue d'explorer les installations de la RCW et découvre par hasard les anciens bâtiments datant des débuts de l'entreprise, fondée à la fin du XIX'e siècle.

Selb y est blessé par une explosion qui déclenche une importante alerte à la pollution (émanations incontrôlées de gaz de chlore). Il réussit à protéger un petit garçon qui jouait près de lui. Ce dernier s'avère être le fils et le petit-fils de deux fidèles employés de l'entreprise. Le grandpère, Heinrich Schmalz, à la retraite, continue d'assurer la surveillance des vieilles installations de la RCW.

Des parasites dans la chaîne stéréo de Selb provoquent un curieux phénomène de rétro-connexion.

Consultés par Selb, Peter Mixkey et Jörg Gremlich ${ }^{18}$, experts du département de surveillance des émissions polluantes du Centre informa-

17. - Cf. note 1. Les traducteurs ont sans doute essayé de rendre une certaine progression avec la suite brouillard - hiver - fin.

18. - Dans la version française Mischkey et Grinsche. 
tique régional, lui expliquent le dispositif informatique d'enregistrement et de suivi des alertes aux émissions toxiques. Ils constatent que la RCW minimise voire nie l'incident. Selb apprend que le phénomène produit par sa chaîne stéréo peut se produire et perturber même des systèmes très sophistiqués, malgré toutes les protections vulnérables aux tentatives de sabotage.

Selb découvre que Mixkey est l'amant de Judith Buchendorff, secrétaire particulière de son beau-frère Korten. Le profil de Mixkey lui semble bien plus correspondre au « hacker» recherché que celui du présumé coupable. Il propose à Mixkey, Judith Buchendorff et à une de ses amies une partie de tennis pour le jour même, sur les courts de la $\mathrm{RCW}$. A priori, les réservations sont au complet depuis fort longtemps, mais Mixkey modifie frauduleusement le planning en leur faveur. Dès son arrivée sur le court, Mixkey comprend le piège. Selb fait son rapport à Korten. La RCW décide d'embaucher Mixkey comptant ainsi le neutraliser. L'affaire semble cette fois-ci réellement close. Fin de l'enquête?

Selb part en vacances.

À son retour, il rencontre Judith Buchendorff qui lui apprend la mort de Mixkey dans un accident de voiture inexplicable. Elle lui demande d'enquêter.

Selb se rend une nouvelle fois au Centre informatique régional pour interroger Gremlich qui se montre peu affecté par le décès de son collègue. Selb apprend que Gremlich vient de rembourser un important prêt immobilier. Interrogé, celui-ci finit par admettre qu'il s'est laissé acheter par la RCW que Mixkey avait soumis à un chantage : il allait dévoiler une documentation compromettante sur le travail forcé de scientifiques juifs, dans l'entreprise sous le Troisième Reich.

Avec l'aide de $\mathrm{M}^{\text {me }}$ Buchendorff, Selb retrouve une partie de cette documentation dans les archives de Mixkey. Il tombe sur un article de 1970 décrivant les humiliations que ces scientifiques avaient subies à la RCW entre 1940 et 1945. Il commence une enquête personnelle sur la collaboration de la RCW avec les nazis.

Selb rend visite au vieux juge Beufer qui avait présidé fin 1943/début 1944 un retentissant procès contre les dénommés Dohmke et Tyberg, respectivement scientifique et directeur général de la RCW, accusés de haute trahison ${ }^{19}$ par le chercheur juif, professeur Karl Weinstein. Dohmke avait été exécuté, Tyberg, libéré in extremis par deux mystérieux SS, avait pu passer en Suisse. Selb, à l'époque procureur stagiaire, avait lui-même instruit le procès.

En se rendant sur le lieu de l'accident de Mixkey, Selb apprend qu'une camionnette «bizarre » y avait été vue avant le drame.

Une nouvelle enquête est confiée à Selb : Sergeï Mencke, danseur professionnel, est soupçonné de s'être automutilé dans l'intention d'escroquer l'assurance. Cette enquête donne à Selb l'occasion

19. - Dohmke et Tyberg auraient dissimulé des découvertes indispensables pour gagner la guerre afin de précipiter la défaite allemande. 
de se rendre aux États-Unis où réside $\mathbf{M}^{\text {me }}$ Hirsch, compagne de Karl Weinstein. Survivants des camps, les deux avaient émigré aux États-Unis où Weinstein avait fait carrière à Stanford. Selb apprend que l'un des SS qui avait libéré Tyberg était Korten dont Tyberg avait plus tard favorisé la carrière à la RCW.

Selb clôt l'enquête sur Menckel.

Selb et Judith Buchendorff (qui a entretemps quitté la RCW) se rendent à Locarno où Tyberg réside depuis sa retraite. Les ultimes pièces du puzzle finissent par se mettre en place : Dohmke et Tyberg étaient innocents et accusés sur ordre par Weinstein. Korten avait parié sur Tyberg pour garantir son avenir professionnel, stratégie qui s'était avérée payante puisque la protection de Tyberg l'avait propulsé au sommet de l'entreprise après 1945. Selb comprend que dans ce jeu de dupes, il n'avait été qu'un simple pion.

Les trois enquêtes convergent et culminent alors dans la plus importante, celle qui touche directement à la vie de Selb et à sa fascination pour Korten qui remonte à leur jeunesse commune:

Au début, je l'ai envié. C'était au Lycée Frédéric-Guillaume à Berlin. Je portais les costumes de mon père, je n'avais pas d'amis et j'étais incapable de monter à la barre fixe. Il était le premier, même en gymnastique, on l'invitait à tous les anniversaires, et les enseignants étaient sérieux quand ils le vouvoyaient. Parfois le chauffeur de son père venait le chercher avec sa Mercedes. Mon père travaillait aux Chemins de Fer du Reich ; en 1934, il avait été muté de Karlsruhe à Berlin.

Korten ne supportait pas l'inefficacité. Il m'a appris à monter à la barre et à en faire le tour. Je l'admirais. Il m'a également montré comment on fait avec les filles. Moi, je courais bêtement à côté de la petite qui habitait l'étage du dessous et qui allait au Luisen, en face de notre lycée. Je l'adulais. Korten, lui, embrassait au cinéma.

Nous sommes devenus amis, nous avons fait nos études ensemble, lui en économie, moi en droit; les portes de sa villa au bord du Wannsee m'étaient ouvertes. Lorsque j'ai épousé sa sœur, Klara, il était témoin et m'a offert la table de travail qui est toujours dans mon bureau, en chêne massif, sculpté, avec des poignées en laiton.

J'y travaille rarement aujourd'hui. Ma profession ne me laisse pas le temps de m'assoir, et lorsque je repasse au bureau en fin de journée, les dossiers ne s'empilent pas sur ma table. Seul le répondeur m'attend et m'indique dans sa petite fenêtre le nombre de messages reçus. Alors je m'installe devant le plateau vide, joue avec un crayon et écoute ce que je dois faire et pas faire, ce que je dois prendre en main et ce à quoi je ferais mieux de ne pas toucher. Je n'aime pas me brûler les doigts. Mais il arrive qu'on se les coince dans le tiroir d'un bureau qu'on n'a pas ouvert depuis longtemps. (Brouillard, p. 11-12. Nous soulignons)

Selb finira effectivement par se « coincer les doigts » dans ce fameux bureau, gage supposé de l'amitié de Korten. Quand il lui apporte la vraie 
solution de l'enquête sur le sabotage du système informatique de la RCW, son beau-frère l'accueille avec « le ton de l'oncle saluant le pénible neveu criblé de dettes qui vient quémander de l'argent » (Brouillard, p. 116). Selb, interloqué, se fera traiter de naïf, de « bonne âme » (ibid., p. 117), quand il recommande de s'attacher les services de Mixkey plutôt que de le poursuivre - un conseil qui sera pourtant suivi.

Mais une fois de plus, le roman n'est pas fini : après avoir démasqué un faux coupable et identifié le vrai, la question de l'accident de Mixkey, et avant tout celle concernant les événements de 1943/44 (donc du passé) resurgissent au moment même où le capitaine d'industrie Korten s'apprête à être honoré par son pays ${ }^{20}$. Le roman continue donc :

Selb découvre la « camionnette bizarre » dans les vieux bâtiments surveillés par Heinrich Schmalz : un savant dispositif de feuilles d'aluminium réfléchissantes fixées sur les portes arrière avait provoqué l'éblouissement qui avait fait perdre à Mixkey le contrôle de sa voiture. C'était donc bien un meurtre. Korten interdit à Selb de continuer à enquêter. Le vieux Schmalz meurt fort opportunément. Les bâtiments qui avaient abrité la camionnette sont rasés. Il n'y a donc plus ni témoin ni preuves.

À l'enterrement de Schmalz, Selb apprend que celui-ci, totalement dévoué à Korten et à l'entreprise, avait été le deuxième SS impliqué dans la libération de Tyberg. C'est aussi Schmalz qui s'était chargé d'éliminer Mixkey, sur ordre de Korten, pour protéger la RCW. Nouvelle fin potentielle, les deux dernières questions ayant trouvé leur réponse.

Selb prend conscience que le procès Tyberg/Dohmke avait été « un complot dans lequel j'avais joué le rôle de l'idiot de service. Préparé et exécuté par mon beau-frère. [...]. Il m'avait utilisé de façon magistrale. [...]. Moi, la bonne âme. » (Brouillard, p. 321) tout comme pour l'enquête sur le sabotage industriel. Comme l'annonce le titre, il décide de faire justice lui-même. Il se rend dans la propriété de Korten en Bretagne où il avait été invité à passer Noël. Korten confirme tout ce que son beaufrère a deviné, reconstruit et reconstitué au fil des différentes enquêtes, mais ne se sentant nullement coupable pense qu'il pourra une nouvelle fois manipuler son beau-frère. Selb pousse alors Korten de la falaise qui surplombe la baie de Trefeuntec. Il repart sans avoir été remarqué. Il faut plusieurs jours pour que la tragique mort « accidentelle » de Korten soit relatée dans les journaux allemands.

20. - Se méprenant sur les vraies motivations de Korten, et pensant le servir une fois encore, Tyberg avait déclaré : «Korten est plus modeste qu'il n'en a l'air. Il a fortement insisté pour que je ne parle pas du rôle qu'il a joué dans ma fuite. J'ai toujours respecté son vœu, pas seulement parce que c'était un geste de modestie, mais aussi de sagesse. Toute cette opération s'accordait mal avec l'image de chef d'entreprise qu'il essayait de se donner. J'ai attendu cet été pour lever le voile sur ce secret. Aujourd'hui la position de Korten comme chef d'entreprise est reconnue de tous et je pense qu'il se réjouira lorsque cette histoire sera publiée au printemps prochain dans le portrait que Die Zeit veut faire paraître à l'occasion de ses soixante-dix ans. » (Brouillard, p. 302). 
Reste à savoir si le détective Selb devenu assassin à son tour sera puni comme dans tout policier qui se respecte. Schlink nous réserve alors un dernier petit suspense. Le roman finit sur la soirée que le détective organise pour fêter le Nouvel An avec ses amis. L'ambiance est joyeuse. Soudain, un coup de sonnette. Devant la porte, un policier en uniforme. Selb se croit perdu et s'apprête à le suivre. Mais coup de théâtre : les voisins se sont juste plaints du bruit...

\section{Selb en quête de lui-même}

Dans un roman policier, l'enquêteur est en général du côté de la justice officielle ou collabore avec elle. L'affaire se complique avec Selb qui avait lui-même représenté l'ordre - la justice - en tant que magistrat à une époque critique de l'Histoire allemande, mais qui avait choisi en 1945 de s'établir comme détective privé :

À la fin de la guerre, on a plus voulu de moi. J'avais été un nationalsocialiste convaincu, membre actif du parti, un procureur impitoyable qui avait aussi demandé et obtenu la peine de mort à plusieurs reprises. Certains de ces procès avaient été spectaculaires. J'ai cru en tout cela et en mon rôle de soldat du front juridique : blessé dès le début de la guerre, je ne pouvais plus être envoyé au front tout court. [...] Après 1945, je suis d'abord allé vivre dans la ferme de mes beaux-parents, puis j'ai travaillé dans le commerce du charbon, un peu plus tard comme détective privé. Je n'avais plus d'avenir comme procureur. Je ne voyais en moi que le procureur national-socialiste que j'avais été et que je ne pouvais plus être. Ma foi avait disparu. Vous ne pouvez pas vous imaginer à quel point on pouvait croire au national-socialisme. Vous, vous avez grandi avec des connaissances que nous n'avons reçues qu'au compte-gouttes, après 1945. C'est avec ma femme que cela a été particulièrement terrible, elle avait été et était restée une belle blonde nazie. Jusqu'à ce qu'elle se mue en une Allemande élancée du miracle économique. [...] À l'époque de la réforme monétaire, on a commencé à réembaucher des collègues compromis. J'aurais sans doute pu revenir dans le système judiciaire. Mais j'avais vu l'effet que produisaient sur les collègues l'effort de réinsertion et la réinsertion elle-même. Loin de se sentir coupables, ils n'éprouvaient plus que le sentiment d'une injustice - celle de leur renvoi - et considéraient leur réinsertion comme une sorte de réparation. Cela m’a dégoûté. (Brouillard, p. 159-150)

Cette déclaration est le moteur de la trilogie où se mêlent enquêtes et quête de soi. Le passé ne cesse de se rappeler à Selb : l'affaire RCW/ Korten et le passé de l'entreprise dans Brouillard sur Mannheim, la dissimulation par le gouvernement allemand d'un dépôt nazi de gaz toxiques enfoui dans l'idyllique forêt de Viernheim dans Un hiver à 
Mannheim $^{21}$, la question de l'attitude de la population allemande envers les Juifs - dénonciation ou aide - sous le national-socialisme dans La fin de Selb. Dans ce dernier roman, le détective subit d'ailleurs, à Berlin où il dit ne pas être retourné depuis soixante ans (donc depuis l'époque nazie si l'on tient compte de l'année de parution du roman), deux agressions relatives au passé/à son passé. Des skinheads l'obligent à faire le salut nazi avant de le jeter dans le Canal du Landwehr et le lendemain, ce sont les jeunes d'un comité antifasciste qui l'avaient vu faire le salut hitlérien la veille qui l'expédient de leur côté dans l'eau... Dans ce troisième volume, Selb semblait enfin être chargé d'une mission simple : retrouver le nom du bailleur de fonds anonyme pour une brochure consacrée au bicentenaire de la Banque Weller\&Welker, la plus ancienne banque privée du Palatinat. Très vite, Selb découvrira qu'elle avait fourni des fonds pour la création de la RCW en 1872 et se trouve maintenant impliquée dans une vaste entreprise de blanchiment d'argent passant par la «Sorbische Genossenschaftsbank Cottbus » achetée après la réunification. Selb finira par identifier le mystérieux bailleur de fonds, un certain professeur Laban, éminent scientifique juif qui s'était occupé de ses neveu et nièce orphelins après la mort de leur parents sous le régime nazi. Le garçon avait réussi à gagner l'Angleterre. À la fille, Weller\&Welker avaient fourni une nouvelle identité au sein de leur famille. Une générosité susceptible toutefois de compromettre l'héritage de Bertram Welker, actuel propriétaire de la banque, mandataire de Selb et accessoirement soupçonné du meurtre de sa femme Stéphanie née Weller. Démasqué par Selb, coupable même de trois assassinats, la fin justifie pour Welker, comme déjà pour Korten, les moyens :

Le passé, le passé. Je ne supporte plus d'entendre ça. Nos parents nous ont assommés avec leurs souffrances pendant la guerre, leurs actes héroïques pendant la reconstruction et le Miracle économique, les jeunes professeurs avec leurs mythes de 1968. Auriez-vous aussi un mythe à me proposer, vous? Arrêtez-ça. Mon devoir à moi, c'est de trouver comment sortir Weller\&Welker de l'ornière. (Fin, p. 257)

21. - Selb y est sollicité pour enquêter sur la disparition d'une jeune étudiante, Leonore Salger. Prétendue morte par suicide, Selb la retrouve se cachant sous l'identité d'une fille au pair américaine. Il apprend aussi que son mandant n'est pas le père de la jeune fille, mais un membre de la cellule terroriste qui avait organisé l'attentat contre le dépôt de gaz toxiques, et que plusieurs de ses camarades ont disparu dans des « accidents ». Leonore, membre de la cellule, est recherchée par la police. Selb l'aide à quitter le pays, l'amène à Locarno chez Tyberg qui accepte de les héberger une nuit bien qu'il ait identifié la jeune fille. Leonore s'enfuit seule en Espagne, Selb, de retour en Allemagne, est arrêté pour entrave à l'enquête policière officielle et passe quelques jours en prison. Libéré, il réussit à négocier l'abandon des poursuites contre Leonore qui finit dans une clinique psychiatrique. 
Un nouveau projet de justice personnelle est cette fois-ci stoppé par le troisième infarctus de Selb. Quelques pages plus tôt, Schlink avait anticipé cette fin en faisant tirer à son détective le bilan désabusé de ses différentes enquêtes :

Oui, ils étaient comme ça. Le Troisième Reich, la guerre, la défaite, la reconstruction et le Miracle économique : pour eux, ce n'étaient que des circonstances changeantes dans lesquelles ils pratiquaient la même activité - ils multipliaient ce qui leur appartenaient ou ce qu'ils géraient. [...] Tout leur était bon du moment qu'ils agrandissaient leurs entreprises, les enrichissaient et les rendaient plus puissantes. Ils avaient le sentiment de faire ce sans quoi rien d'autre n'existerait. A quoi bon des gouvernements, des systèmes, des idées, les douleurs et les joies de l'être humain, si l'économie ne prospérait pas? (Fin, p. 232)

\section{La justice selon Schlink}

Dans Un hiver à Mannheim, Selb dénonce la parodie de justice à laquelle se livrent les instances de son pays dans le cas de Leonore Salger (cf. note 21), poursuivie pour un attentat qui, officiellement, n'a pas eu lieu parce que toujours officiellement, le dépôt de gaz toxiques n'a jamais existé, et s'il devait exister, il ne serait pas localisé à Viernheim où s'étaient passés les événements, mais à Käfertal... Ce dépôt de Viernheim, organisé par les nazis comme le prouve une carte des lieux portant le tampon de la croix gammée que Selb avait pu se procurer, est considéré comme devant rester secret car susceptible de déclencher la panique dans la population, de perturber les relations germano-américaines - et accessoirement de compromettre un juteux projet de construction immobilière. L'arrestation de Selb se trouvant entachée d'un vice de procédure, il est libéré rapidement. Dans le plus profond non-respect des lois dont il fustige les mensonges, l'hypocrisie et l'inefficacité, il en profitera pour cambrioler l'appartement de l'un des suspects où il trouvera une fois de plus de quoi prouver - à lui-même, puisque la justice ne veut rien savoir - la justesse de ses hypothèses.

Juriste de formation, Schlink avait rappelé en 1988 que l'un des objectifs de la reconstruction démocratique de l'Allemagne avait été de créer une justice impartiale, indépendante, ne pouvant pas être mise au pas $^{22}$. Un bel objectif saboté, à partir de 1948, par la réintégration des anciens magistrats compromis (évoquée par Selb dans Brouillard sur Mannheim) qui avait été lourde de conséquences :

22. - Cf. «Kollektivschuld ?», in : Vergangenheitsschuld, Beiträge zu einem deutschen Thema. Zürich, Diogenes, 2007, p. 11-33. 
Que ceux qui s'étaient compromis dans le Troisième Reich n'aient pas été mis au ban, poursuivis et jugés, mais tolérés, respectés, laissés dans les positions qui avaient été les leurs et même aidés dans leurs carrières, acceptés en tant que professeurs et parents, a eu comme conséquence d'impliquer la génération des parents comme celle des enfants dans les crimes et la culpabilité du Troisième Reich. Pour éviter cette implication, il aurait fallu pratiquer systématiquement la mise à l'écart, engager des poursuites pénales, juger. Mais vu le nombre de criminels et de suiveurs, de personnes qui savaient, se contentaient de regarder ou s'efforçaient de ne rien voir, il n'avait pas été possible de procéder ainsi. ${ }^{23}$

C'est cette impossibilité réelle ou intentionnelle de faire table rase et ses conséquences que Schlink met en scène dans la trilogie en rappelant, à travers les différents cas qu'il résout, l'implication intergénérationnelle qui en découle : la culpabilité, constate-t-il, « persiste dans l'Histoire, et reste vivante dans le futur » 24 . Selb, justicier autoproclamé et atypique, se retrouve ainsi pris dans un jeu de miroirs entre présent et passé 25 , dans un «présent du passé » comme le formule le titre d'un autre article de Schlink où celui-ci revient, en 2001, sur sa propre expérience d'enfant né en 1944 - donc ni responsable ni coupable - et qui avait connu non seulement les débats autour du poids des crimes du national-socialisme, mais aussi l'effet de banalisation, voire de saturation résultant des innombrables actes de contrition plus ou moins sincères dont il met en doute l'impact : «Quand la distance historique est devenue suffisamment grande, nous ne nous sentons plus concernés par ce qui est unique, par ce qui échappe à toute comparaison et par ce qui est passé, et le pathos moralisant dont on use et abuse pour en parler n'atteint plus sa cible »26. Et pourtant, le passé résiste.

23. - « Dass die Täter des Dritten Reiches nicht ausgestoßen, nicht verfolgt und verurteilt, sondern toleriert, respektiert, in ihren Positionen belassen und bei ihren Karrieren gefördert, als Lehrer und Eltern akzeptiert wurden, hat die Generation der Täter und die ihrer Kinder in die Verbrechen und Schuld des Dritten Reiches verstrickt. Aber angesichts der Fülle der Täter und Teilnehmer, Mitwisser, Zu- und Wegschauer war die erforderliche Konsequenz nicht zu leisten. » Cf. « Die Bewältigung von Vergangenheit durch Recht », in : Vergangenheitsschuld, op. cit. p. 80-111. Ici p. 88. Traduction IRM.

24. - «In der Geschichte bleibt die Schuld bewahrt, in ihr bleibt sie in der Zukunft lebendig. » Cf. «Kollektivschuld ? », in op. cit., p. 33. Traduction IRM.

25. - D'un point de vue juridique et mathématique ce n'est que vers 2025, écrit Schlink, que le dernier Allemand pouvant être tenu pour juridiquement responsable des événements avant le 9 mai 1945 aura disparu. Op. cit., p. 11-12.

26. - «Was einmalig, unvergleichbar und vergangen ist, engagiert uns bei ausreichendem historischen Abstand nicht mehr, und das moralische Pathos, mit dem gleichwohl darüber geredet wird, geht ins Leere. » Cf. « Die Gegenwart der Vergangenheit », in: Vergangenheitsschuld, op. cit., p. 12-123, ici p. 114. Traduction IRM. 
Schlink a créé avec Selb non pas un double mais un personnage qui lui permet d'exprimer de manière originale ses doutes sur le passé allemand, sur les derniers acteurs du nazisme, sur la transmission de l'Histoire, sur le fonctionnement de l'appareil judiciaire de l'Allemagne, et plus généralement sur son pays qu'il considère se fourvoyant dans une culture commémorative « diplomatique » plutôt que dans une vraie intégration de son passé. Dans Vergewisserungen, Schlink évoque l'héritage du Troisième Reich dont l'auteur, l'écrivain, l'artiste doit aujourd'hui encore se saisir, s'emparer, quel que soit le genre choisi ${ }^{27}$ : qu'il ait luimême opté pour le roman policier pour mettre en scène la difficile question du passé dans le présent est peut-être une invitation à ses lecteurs à mener l'enquête à leur tour.

27. - « Schlage die Trommel und fürchte dich nicht! », in: Vergewisserungen. Über Politik, Recht, Schreiben und Glauben. Zürich, Diogenes, 2005, p. 181-193. 
Rabenstein-Michel.indd 94 